\title{
In Vivo Imaging and Software Analysis Revealed the Contribution of Endothelial Damage to Thrombus Development Processes
}

\author{
Satoshi Nishimura ${ }^{1,2,3}$ Akira Sawaguchi ${ }^{4}$ \\ 1. Center for Molecular Medicine, Jichi Medical University, Tochigi, Japan \\ 2. Department of Cardiovascular Medicine, the University of Tokyo, Tokyo, Japan \\ 3. Translational Systems Biology and Medicine Initiative, the University of Tokyo, Tokyo, Japan \\ 4. Department of Anatomy, Ultrastructural Cell Biology, Faculty of Medicine, University of Miyazaki, \\ Miyazaki, Japan
}

The cellular mechanisms associated with cardiovascular events remains unclear, largely because of an inability to directly visualize and evaluate thrombus formation in living body. We developed in vivo imaging technique based on multi-photon microscopy and light-manipulation technique to reveal the multicellular processes during thrombus development. Additionally we developed software analysis system which can evaluate thrombotic processes from XYZT visuals, with high reproducibility. The software did not use 'parameter' adjustment by observers, and completely free from researchers bias. We visualized the cell dynamics in single platelet levels, and assessed thrombus formation processes using three animal models. First, we induced rapidly developing thrombi composed of discoid platelets, which was triggered by ROS stimulation by photo-chemical reactions.

In this model, thrombus development was mainly dependent on P-selectin and GPIIbIIIa activations. The functional properties including activation, aggregation, and fibrinogen binding were different between 'newer' and 'aged' platelets in developing thrombus. In the second model, thrombus formation was induced by endothelical cell disruption by laser irradiations. With the rapid recruitment of inflammatory leukocytes into damaged area, fibrin net formation and tissue regenerative changes were also observed. TLR4 signaling contributed to these steps, and pretreatmet of LPS markedly enhanced inflammatory reactions. In last model, spontaneous platelet aggregations were induced by transient ischemia and reperfusions.

We revealed that the endothelial cell damage levels strictly determine the entire course of thrombotic processes, and our imaging system can evaluate the therapeutic strategies against them. Automatic software analysis can be powerful tools to give statistical evidence from XYZT visuals.

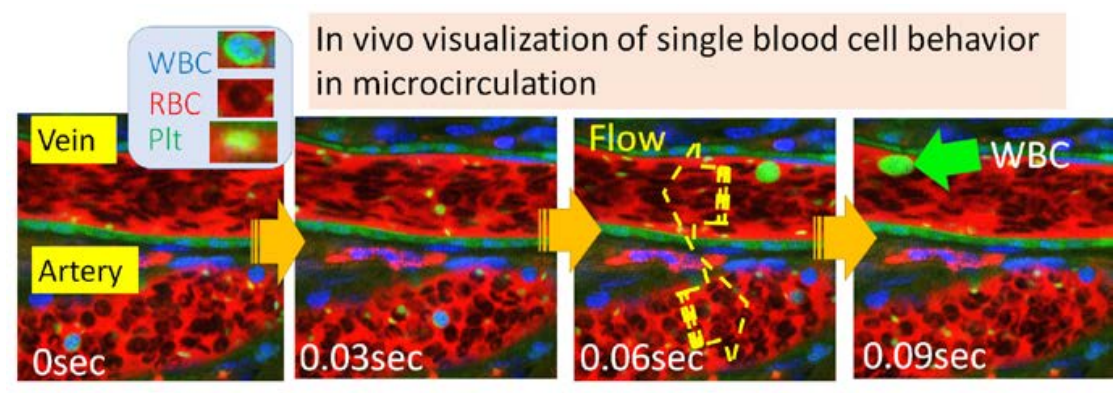



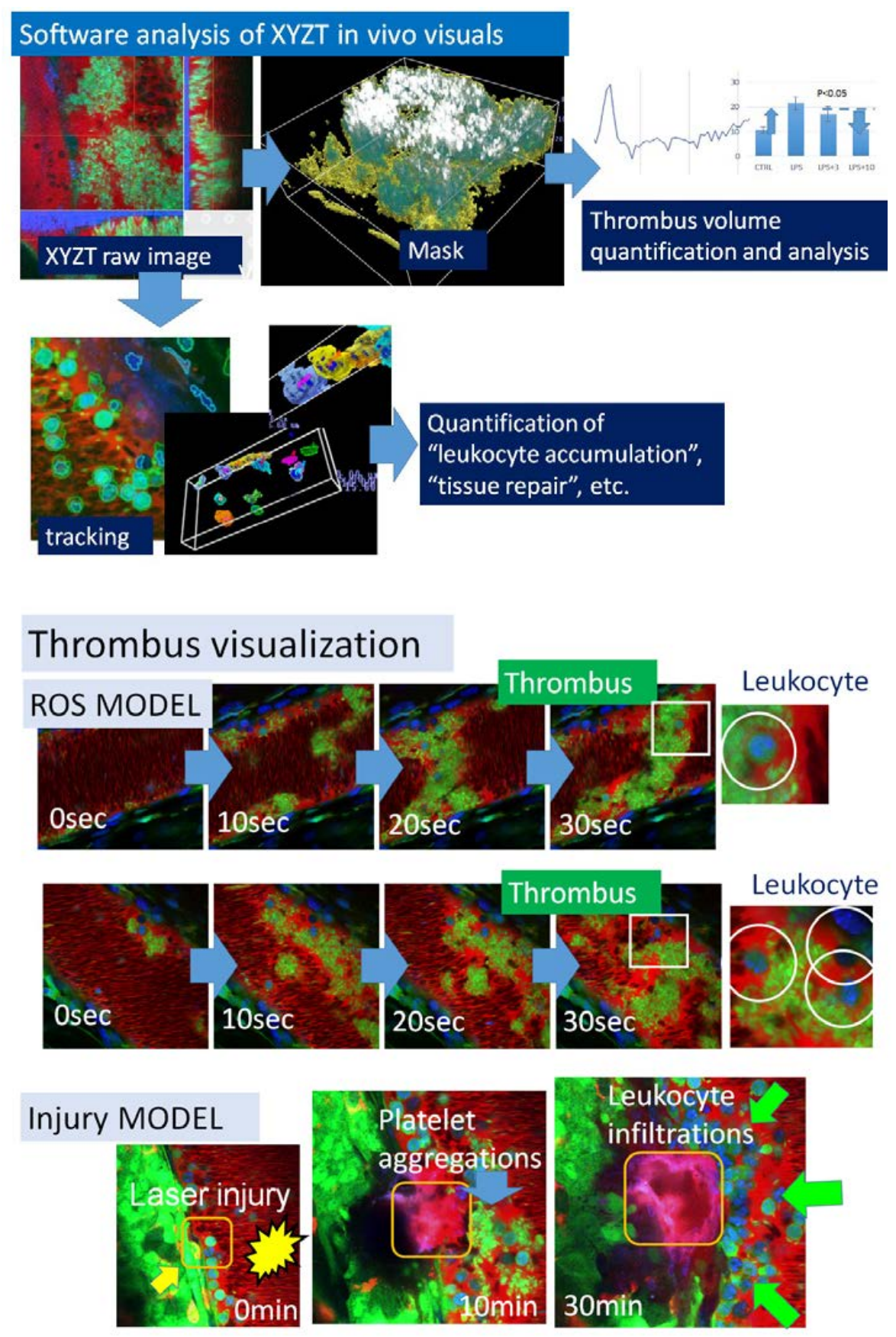

Figure 1

References:

[1] Nishimura S, et al. Cell Metabolism. 2013, 18, 759-766

[2] Nakamura S, Takayama N, ,,,, Nishimura S, Eto K Cell Stem Cell, 2014 in publication.

[3] Nishimura S, et al. Blood. 2012 ;119(8):e45-56.

[4] Takayama N, Nishimura S, et al. J Exp Med, 2010; 207(13):2817-2830

[5] Takizawa H*, Nishimura S* $^{*}$, et al. (*equal contribution) J Clin Invest., 2010, 120(1): 179-190.

[6] Nishimura S, et al. Nature Medicine, 2009, 15:8, 914-920.

[7] Nishimura S, S. et al. Progress in Biophysics and Molecular Biology, 2008;97:282-297.

[8] Nishimura S, et al. J Clin Invest. 2008, 118(2): 710-721.

[9] Nishimura S, et al. Diabetes. 2007,56:1517-1526. 